\title{
Study on a bottom topography of a lake using positioning data and depth data
}
K. Yamamoto ${ }^{1}$
Hashentuya $^{2}$
Y. Otani ${ }^{3}$
Y. $\mathrm{He}^{4}$

\section{Watanabe 5}

(Received 9 January 2012; revised 15 October 2012)

\begin{abstract}
The bottom topography of a lake is investigated. Numerical techniques to utilize positioning data and depth data are described. Data sets obtained in RTK-GPS positioning consist mainly of temporal, latitudinal, longitudinal, and altitudinal records. Data sets obtained in echo-sounder measurement consist of temporal records and depths. The latitudinal and longitudinal data are transformed to rectangular coordinates by the Gauss-Krüger Projection, and the resultant positional data sets are synchronized with depths to yield trajectories over the lake's floor. A bottom topography over a triangular mesh is updated using the positioning data and the depth data.
\end{abstract}

http://journal.austms.org.au/ojs/index.php/ANZIAMJ/article/view/5110 gives this article, (c) Austral. Mathematical Soc. 2012. Published November 5, 2012. ISSN 1446-8735. (Print two pages per sheet of paper.) Copies of this article must not be made otherwise available on the internet; instead link directly to this URL for this article. 


\section{Contents}

1 Introduction

C526

2 Positioning and depth measurement

C528

3 Synchronization of positioning and depth data

C533

4 Conclusion

C535

References

C537

\section{Introduction}

Seto Inland Sea divides the main island and the Shikoku Island of Japan. Kojima Lake is located on the coast of the Seto Inland Sea. Kojima Bay is connected to the Seto Inland Sea. A portion of the Kojima Bay was isolated by an embankment and became a reservoir called Kojima Lake. Kojima Lake is surrounded by two municipalities, Okayama and Tamano in the Okayama Prefecture. It is approximately $10 \mathrm{~km}^{2}$ in area. The water level of the lake is controlled to maintain its average depth ranging from approximately $1.8 \mathrm{~m}$ to $2.1 \mathrm{~m}$. The primary water source for the Kojima Lake is the supply from two rivers, the Kurashiki River and the Sasagase River [1]. There are six gates located on the bank. Those gates are opened when it is necessary to discharge water from the Kojima Lake to the Kojima Bay to maintain the water level of the lake. In this study, the bottom topography of the Kojima Lake is investigated by a RTK-GPS and a high precision echo sounder. Figure 1 shows hardware used in our measurements [3]. Figure 1(a) shows the antenna of the RTK-GPS rover station, which is connected to the echo sounder sensor unit underneath the surface of the water. Figure 1(b) shows the RTK-GPS base station set on the ground. 


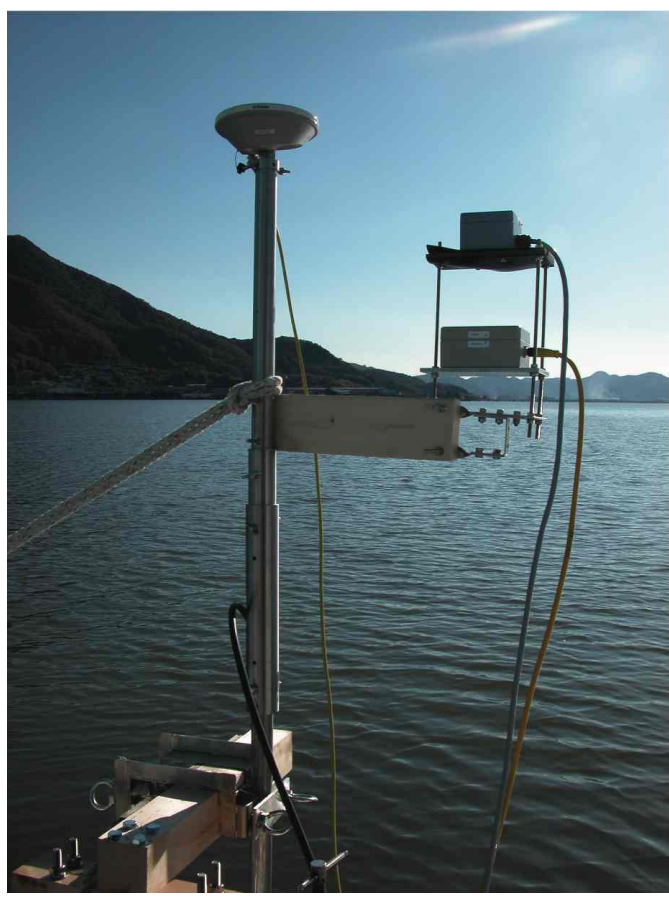

(a) The rover antenna attached to the top of a pole. The sensor unit of an echo sounder is attached to the other end of the pole underneath the surface of the water.

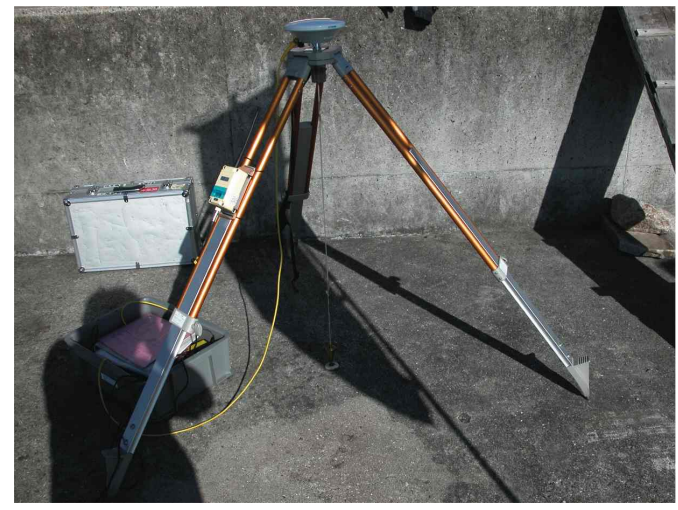

(b) Outcomes from the base station are transmitted to the rover station through the wireless transmitter.

Figure 1: Hardware configuration [3]. 


\section{Positioning and depth measurement}

In the previous study [3], measurements using a RTK-GPS and an echo sounder were conducted on September 19th, 21st, 22nd, 26th, November 20th, December 23rd, 2010, and January 8th, 2011. Measurements were also conducted on April 14th, 20th, May 15th, and November 26th, 2011. In this study we present results in which new outcomes from the measurements on and after April 14th are introduced into an analysis together with those obtained in the previous study.

Data sets obtained in RTK-GPS positioning consist of latitudinal, longitudinal, and altitudinal data recorded at every second. Longitudinal and latitudinal records are transformed with the Gauss-Krüger Projection to yield trajectories on the floor of the lake. Figures $2-5$ show results of the measurements conducted on April 14th, April 20th, May 15th, and November 26th. Outcomes from the RTK-GPS positioning include temporal records and positional records to determine the position of the antenna of the rover station at given times. RTK-GPS positioning data given in terms of latitude and longitude were transformed to rectangular coordinates by the Gauss-Krüger Projection. Figure 2(a), (b), (c) and (d) shows the wakes of the antenna in the $x y$-plane with the outline of the Kojima Lake on April 14th, April 20th, May 15th, and November 26th, respectively.

Figure 3 shows enlargements of Figures 2. Figure 4 shows the data obtained in echo-sounder measurements. The positional data shown in Figure 2 are synchronized with the depth shown in Figure 4 to generate the trajectories on the floor of the lake, shown in Figure 5. 


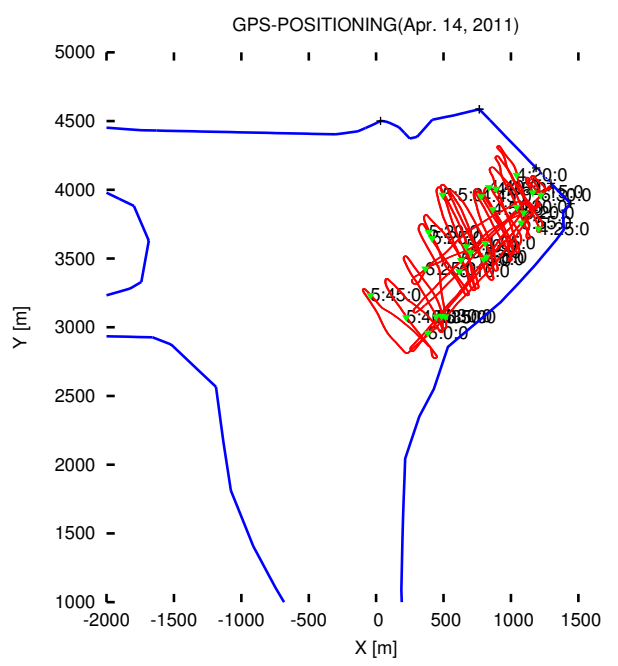

(a) April 14th, 2011

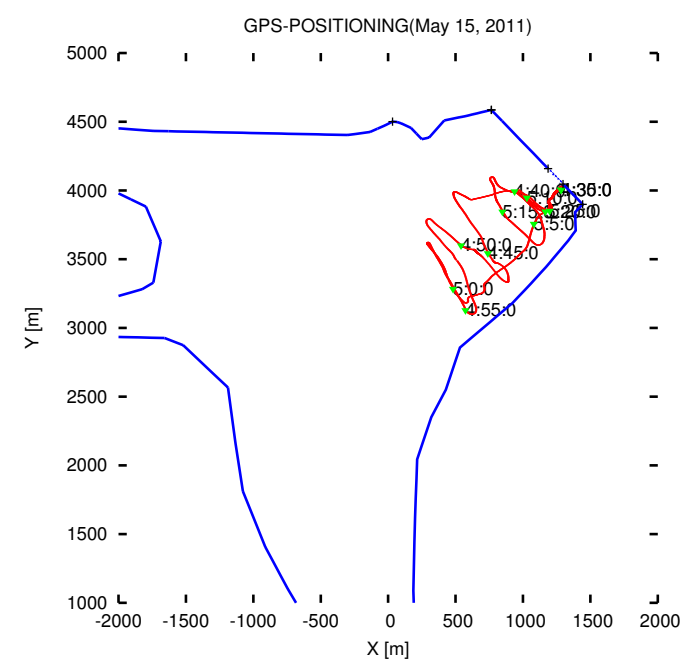

(c) May 15th, 2011.

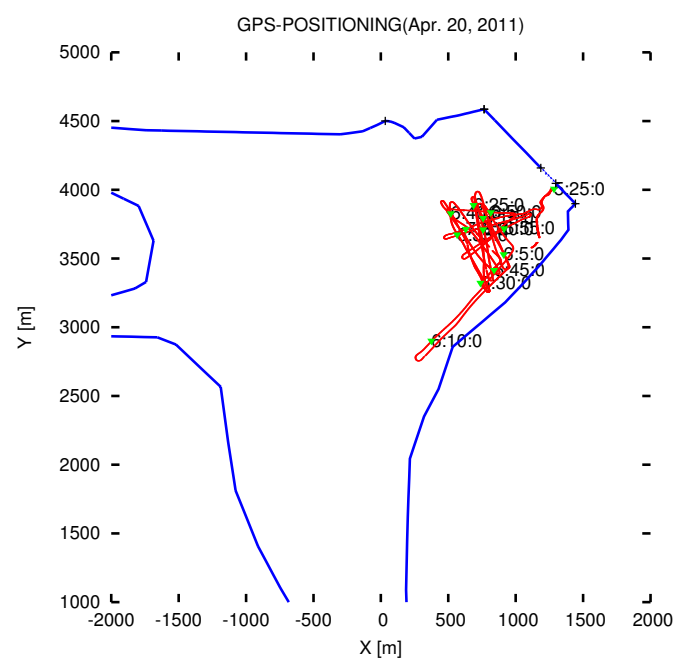

(b) April 20th, 2011.

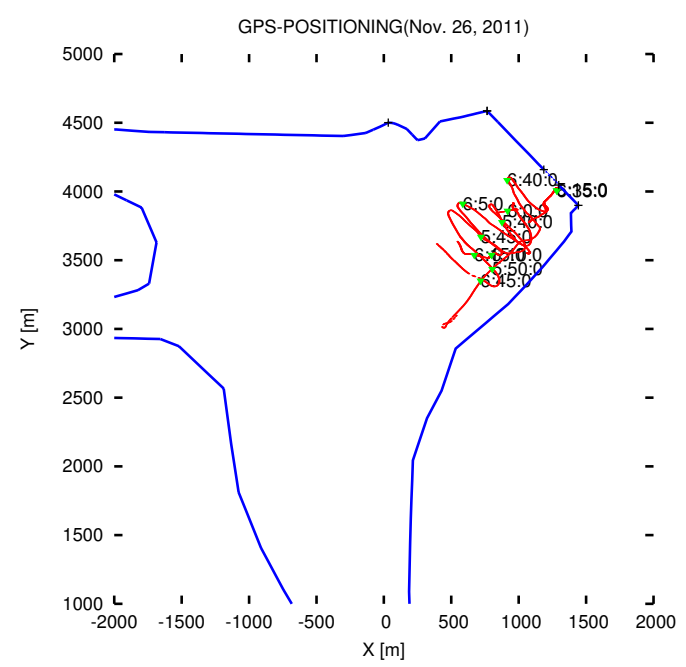

(d) November 26th, 2011.

Figure 2: Wakes of the rover antenna and the time are indicated with an outline of the Kojima Lake. 


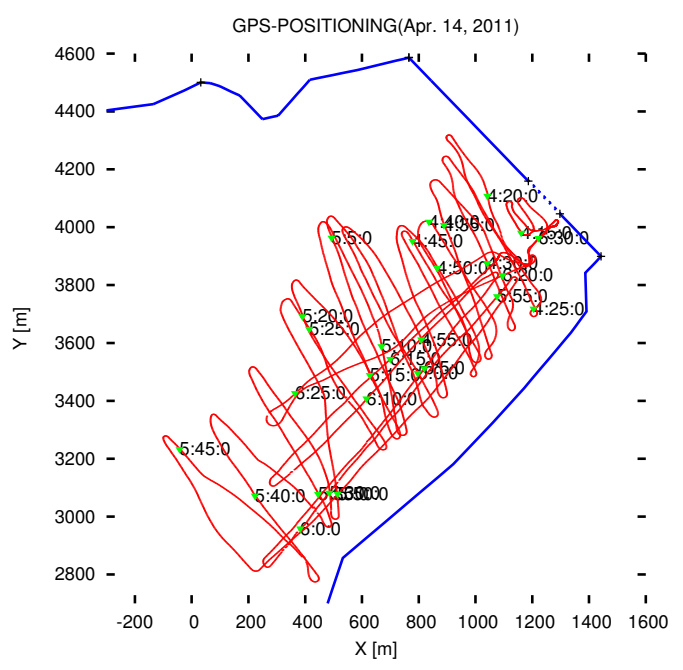

(a) April 14th, 2011

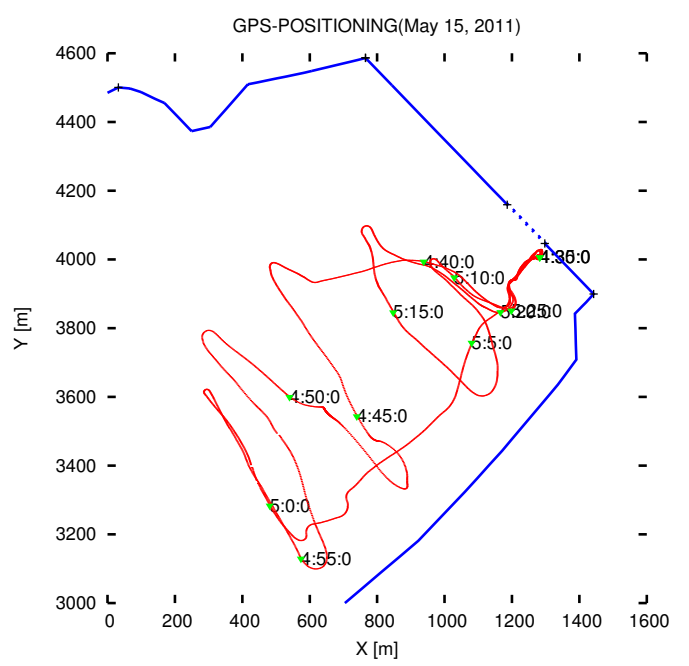

(c) May 15th, 2011.

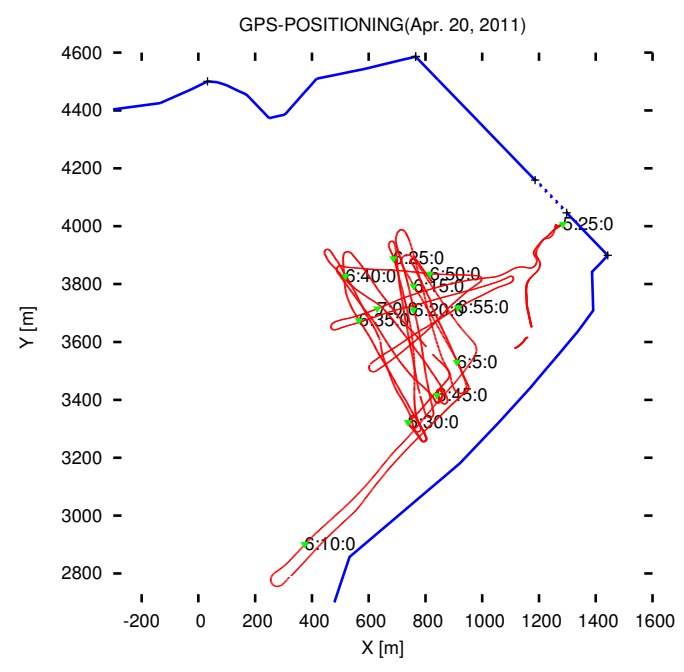

(b) April 20th, 2011.

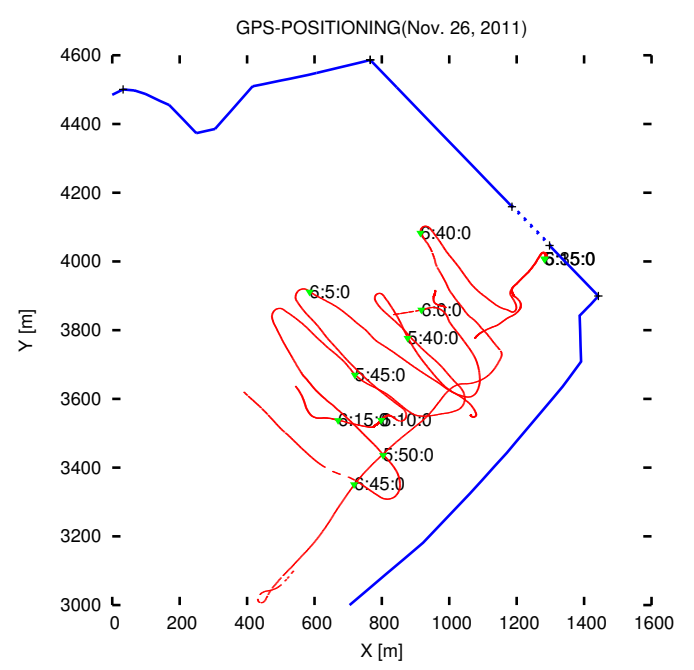

(d) November 26th, 2011.

Figure 3: Enlargements of parts containing the wakes of the rover antenna in Figure 2 and the gates on the embankment between the Kojima Lake and the Kojima Bay in the rectangle $[1200,1400] \times[4000,4200]$. 

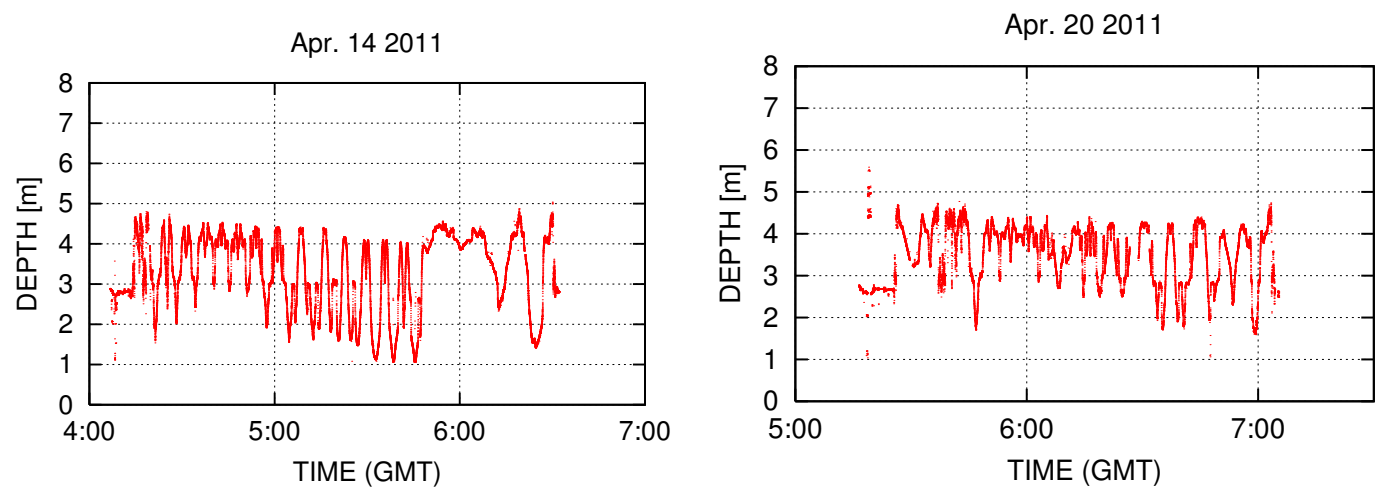
(a) April 14th, 2011
(b) April 20th, 2011.
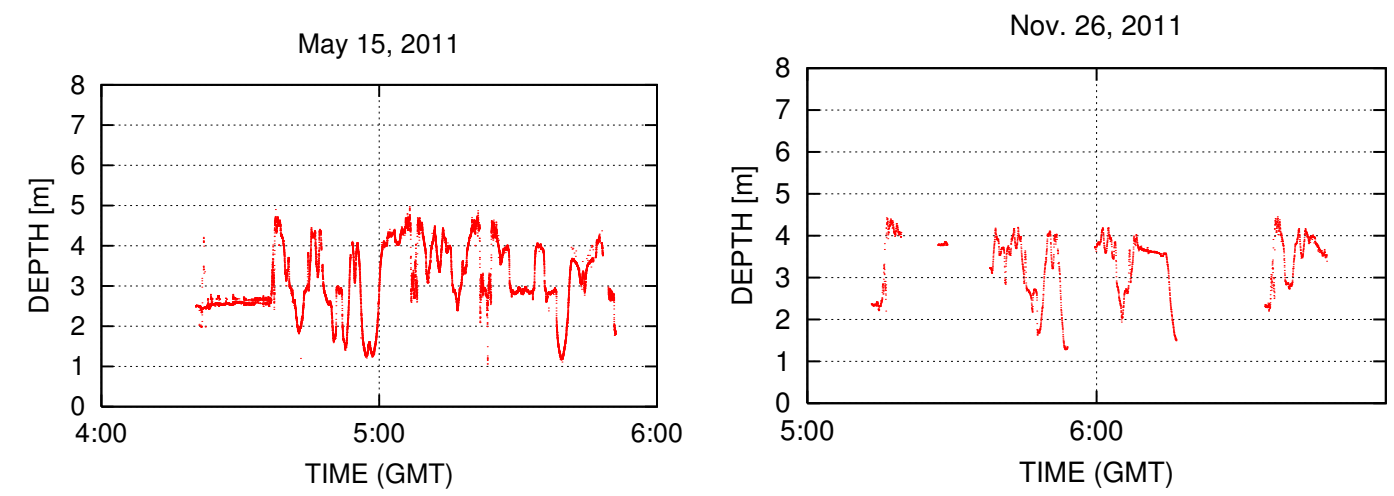

(c) May 15th, 2011.

(d) November 26th, 2011.

Figure 4: Graph of the depth as a function of time. 


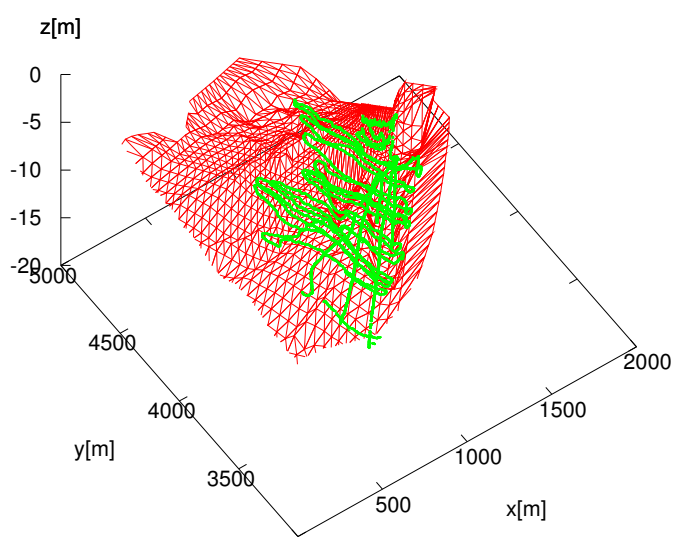

(a) April 14th, 2011

3-D(May 15, 2011)

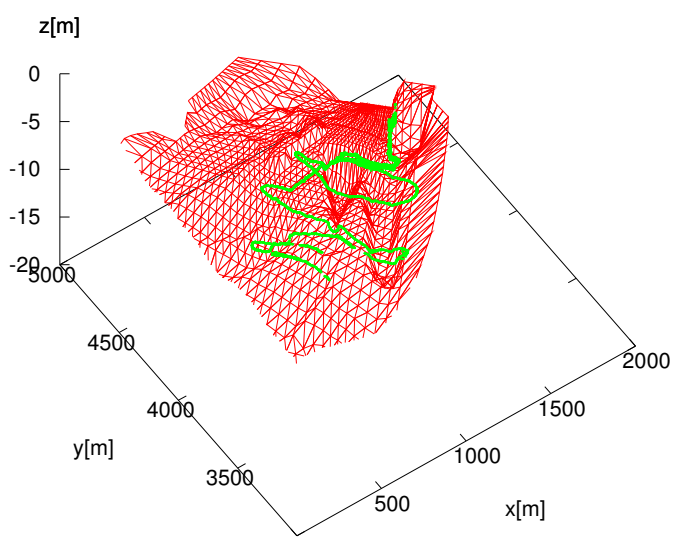

(c) May 15th, 2011.

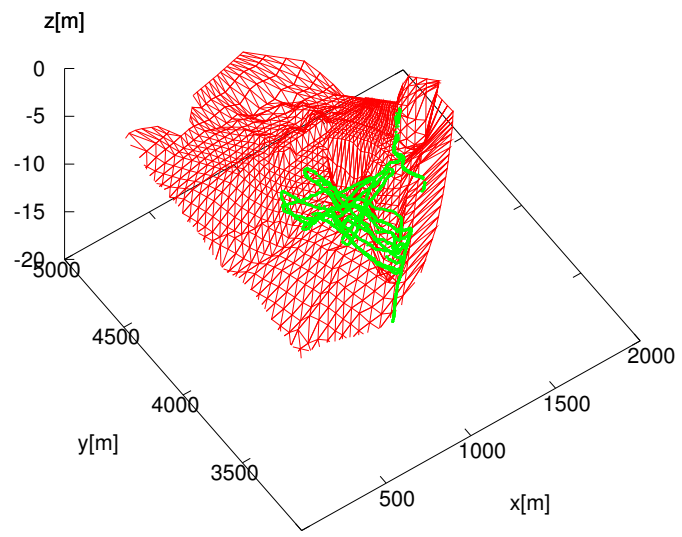

(b) April 20th, 2011.

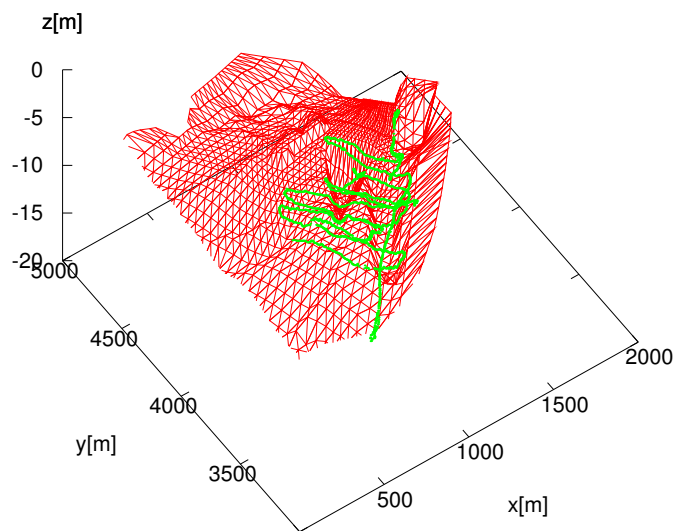

(d) November 26th, 2011.

Figure 5: The positional data of the rover antenna and the depth are synchronized to generate the projected trajectory of the wake of the positioning unit over the floor of the Kojima Lake. The topography before the update is also shown for comparison. 


\section{Synchronization of positioning and depth data}

Time-position data and time-depth data were synchronized to yield depthposition data. Results are shown in Figures 2-5. Previous bottom topography over a finite element mesh shown in Figure 6(a) is also indicated in those figures. The data from the measurements were introduced into analysis to update the bottom topography. The results are shown in Figures 6-8. Figures 6(a) and 6(b) show wire frames of bottom topographies before and after the update, respectively.

In order to generate the updated bottom topography, the depth data are first used to update the topography on each element in the finite element mesh. The depth data of each node is the average of the depth determined by the elements which contain the node. Let $E$ be an triangular element with vertices $V_{1}\left(X_{1}, Y_{1}\right), V_{2}\left(X_{2}, Y_{2}\right)$ and $V_{3}\left(X_{3}, Y_{3}\right)$. Let $z_{1}, z_{2}, \ldots, z_{n}$ be depth data at $\left(x_{1}, y_{1}\right),\left(x_{2}, y_{2}\right), \ldots,\left(x_{n}, y_{n}\right)$ contained in $E$. The bottom topography is approximated by a linear function $z=a x+b y+c$ on each triangular element. The parameters are obtained by a least square approximation. Suppose $a, b$ and $\mathrm{c}$ minimize

$$
s=\sum_{k=1}^{n}\left[z_{k}-\left(a x_{k}+b y_{k}+c\right)\right]^{2} .
$$

Then $a, b$ and $c$ are obtained by solving the system of linear equations

$$
\frac{\partial s}{\partial a}=0, \quad \frac{\partial s}{\partial b}=0, \quad \frac{\partial s}{\partial c}=0,
$$


or

$$
\begin{aligned}
& a \sum_{k=1}^{n} x_{k}^{2}+b \sum_{k=1}^{n} x_{k} y_{k}+c \sum_{k=1}^{n} x_{k}-\sum_{k=1}^{n} x_{k} z_{k}=0, \\
& a \sum_{k=1}^{n} x_{k} y_{k}+b \sum_{k=1}^{n} y_{k}^{2}+c \sum_{k=1}^{n} y_{k}-\sum_{k=1}^{n} y_{k} z_{k}=0, \\
& a \sum_{k=1}^{n} x_{k}+b \sum_{k=1}^{n} y_{k}+n c-\sum_{k=1}^{n} z_{k}=0 .
\end{aligned}
$$

Let $V\left(X_{0}, Y_{0}\right)$ be a node in the finite element mesh. Suppose that $V$ is a vertex of elements $E_{1}, E_{2}, \ldots, E_{m}$. Suppose that the bottom topography over the kth element is represented by the linear function $z=a_{k} x+b_{k} y+c_{k}$. Then $Z_{V}$, the updated depth at $V$, is the weighted average of $z_{1}, z_{2}, \ldots, z_{m}$ with respect to the areas of $E_{1}, E_{2}, \ldots, E_{m}$,

$$
Z_{V}=\frac{\sum_{k=1}^{m} z_{k} A_{k}}{\sum_{k=1}^{m} A_{k}},
$$

where $z_{k}=a_{k} X_{0}+b_{k} Y_{0}+c_{k}$ and $A_{k}$ is the area of $E_{k}$.

Figures $7(\mathrm{a})$ and $8(\mathrm{a})$ show bottom topographies over the entire region before and after the update, respectively. Figures 7(b) and 8(b) show bottom topographies over a region near the gates before and after the update, respectively.

The colour variation from blue to red corresponds to the depth range from $-6 \mathrm{~m}$ to $0 \mathrm{~m}$. Figure $7(\mathrm{~b})$ shows the existence of extremely deep areas with depth more than or equal to $-6 \mathrm{~m}$ near the gates before the update. Figure 8 (b) shows that those areas have disappeared after dredging constructions and filling constructions. 


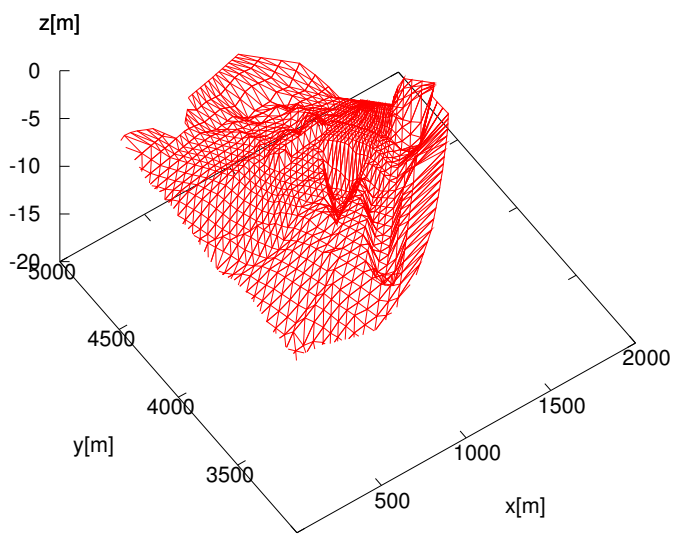

(a) Wire frame before update [3].

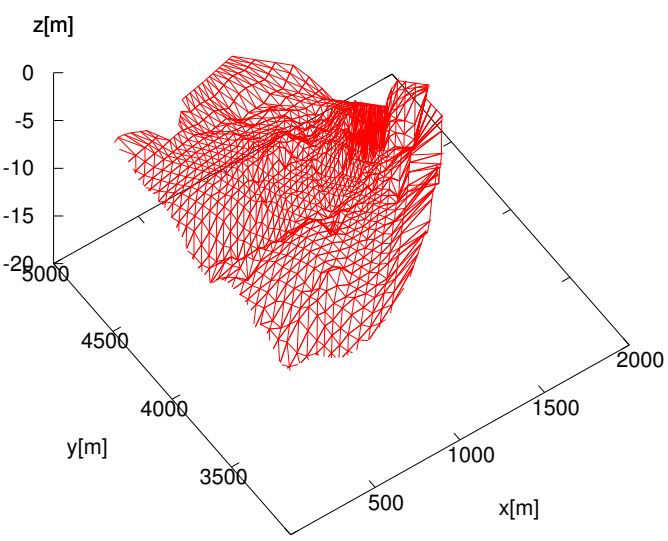

(b) Wire frame after update.

Figure 6: Wire frames before and after update: The figure shows the wire frames for the bottom topography before the update and after the update using the data shown in Figures 2-5 and the data obtained in the previous study [3].

\section{Conclusion}

What is novel about our techniques is that RTK-GPS positioning data and echo sounder data are implemented to update the bottom topography over a finite element mesh, and that a three dimensional update bottom topography is generated by synchronizing one dimensional RTK-GPS data and echo sounder data. We described techniques to update the bottom topography of a lake. Figures 7-8 show significant changes in the bottom topography due to sedimentation. Accurate data are required for analysis of flow generated by the discharge of water from the lake [1,2], and our results show that frequent updates of the bottom topographical data are necessary. 


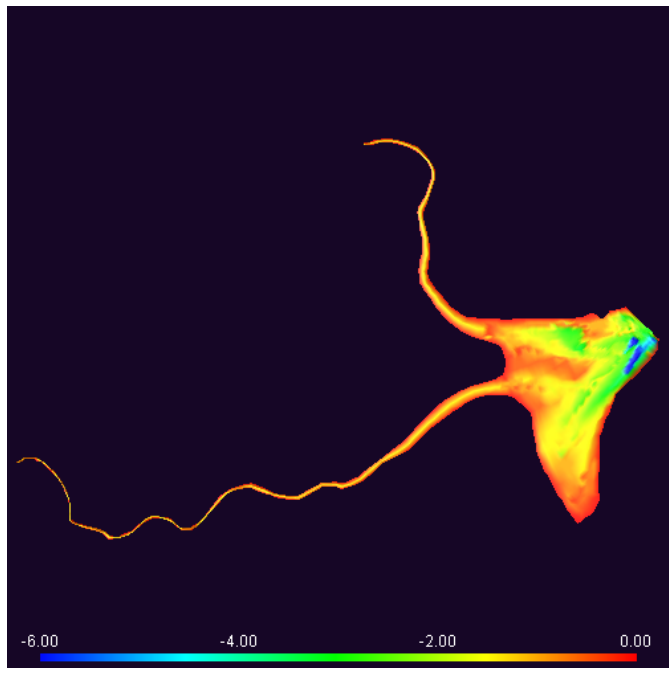

(a) Previous bottom topography of entire region [3].

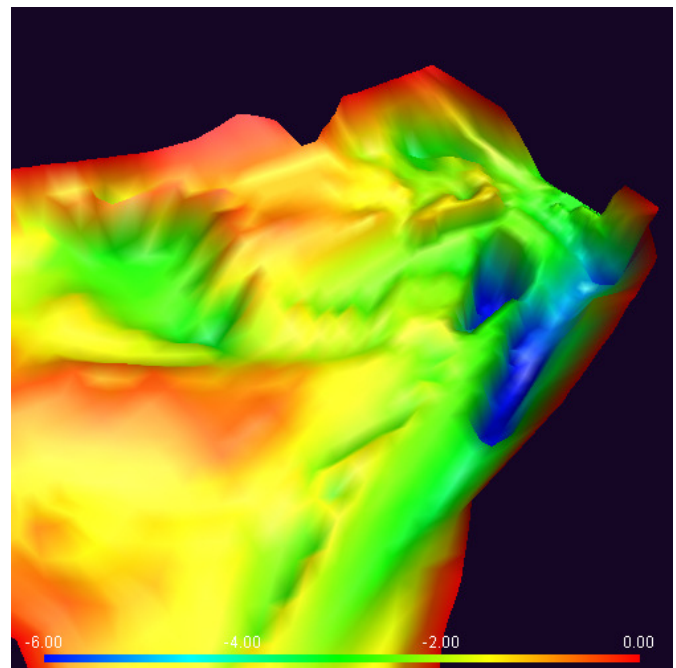

(b) Previous bottom topography near the gates.

Figure 7: Previous bottom topography.

Figure 1(a) shows a digital biaxial clinometer and electronic compass which calibrate the outcomes from the RTK-GPS and the echo-sounder and correct the error due to the movement of the boat such as pitch, roll, and yaw. This technique was utilized in the measurement conducted on November 26th, 2011 to obtain the results shown in Figures 2-5(d).

Acknowledgements We are grateful to the Bay Central Administration Office, the Section of Land Improvement in the Kojima Bay Area, the Okayama Prefecture Okayama Development Bureau, the Engineering Workshop, Center for Engineering Innovation, the Faculty of Engineering, Okayama University, and those people who assisted in the field measurement. The information for generating figures concerning the Kojima Lake is partially based on maps (C)Shobunsha Publications, INC., (C)Nihon Computer Graphic CO., LTD. This study was supported by the Wesco Scientific Promotion Foundation. 


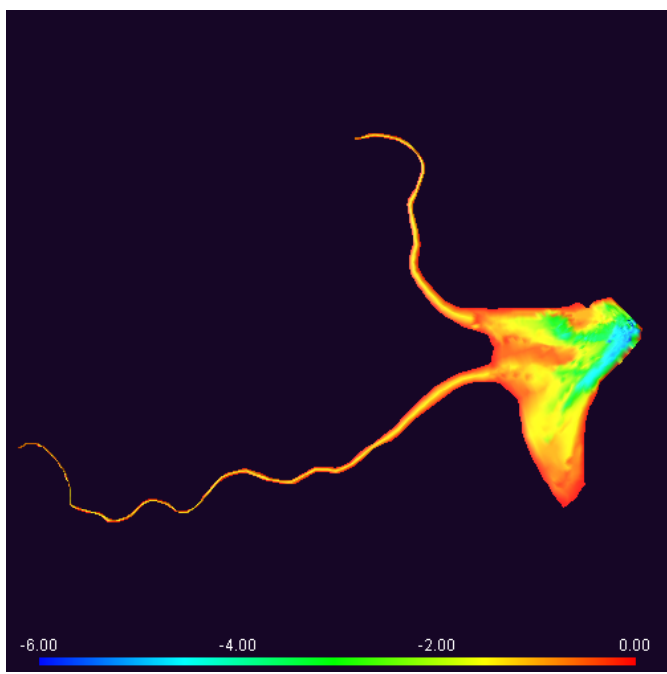

(a) Updated bottom topography of entire region.

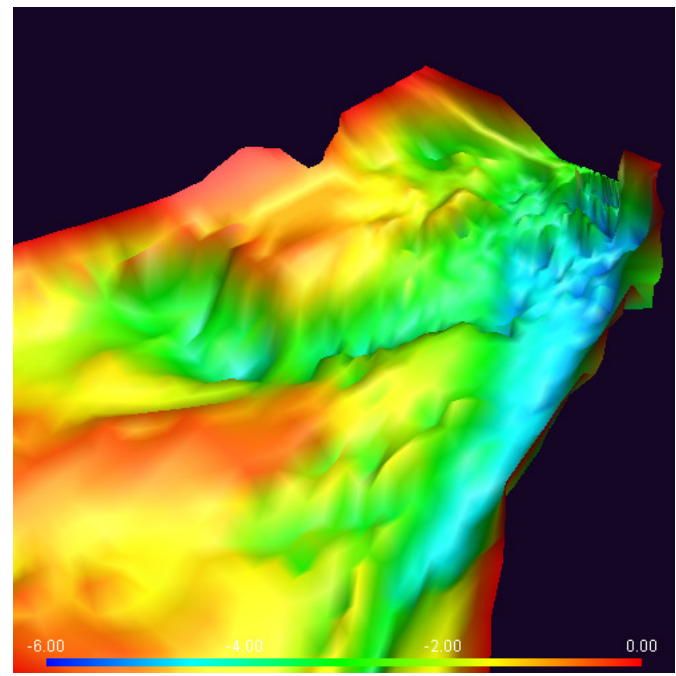

(b) Updated bottom topography near the gates.

Figure 8: Updated bottom topography.

\section{References}

[1] Shinya Sumida, Ying Liu, Yu Li, Kazuhiro Yamamoto, Majda Ceric, Masaji Watanabe, Numerical study of currents generated in a lake, and verification by experiment, ANZIAM J., 49:C541-C558, May 2008. http://journal . austms.org.au/ojs/index.php/ANZIAMJ/article/ view/365 C526, C535

[2] Ying Liu, Kazuhiro Yamamoto, Majda Ceric, Masaji Watanabe, Numerical study of flow generated in a lake and experiment using GPS, ANZIAM J., 50:C912-C929, June 2009. http://journal .austms org. au/ojs/index.php/ANZIAMJ/article/view/1470 C535

[3] Kazuhiro Yamamoto, Hashentuya, Yoji Otani, Masaji Watanabe, Bottom Topography Measurement using RTK-GPS and High Precision 
Echo Sounder, CICC-ITOE 2011. Volume 2:309-312,2011 C526, C527, C528, C535, C536

\section{Author addresses}

1. K. Yamamoto, Graduate School of Environmental Science Okayama University, 1-1, Naka 3-chome, Tsushima, Kita-Ku Okayama 700-8530, JAPAN.

2. Hashentuya, Graduate School of Environmental Science Okayama University, 1-1, Naka 3-chome, Tsushima, Kita-Ku Okayama 700-8530, JAPAN.

3. Y. Otani, Graduate School of Environmental Science Okayama University, 1-1, Naka 3-chome, Tsushima, Kita-Ku Okayama 700-8530, JAPAN.

4. Y. He, Graduate School of Environmental Science Okayama University, 1-1, Naka 3-chome, Tsushima, Kita-Ku Okayama 700-8530, JAPAN.

5. M. Watanabe, Graduate School of Environmental Science Okayama University, 1-1, Naka 3-chome, Tsushima, Kita-Ku Okayama 700-8530, JAPAN.

http://www. okayama-u.ac.jp/

mailto: watanabe@ems.okayama-u.ac.jp 